\title{
SHOALING OF NONLINEAR INTERNAL WAVES ON A UNIFORMLY SLOPING BEACH
}

\author{
Kei Yamashita ${ }^{1}$, Taro Kakinuma ${ }^{2}$ and Keisuke Nakayama ${ }^{3}$
}

\begin{abstract}
The internal waves in the two-layer systems have been numerically simulated by solving the set of nonlinear equations in consideration of both strong nonlinearity and strong dispersion of waves. After the comparison between the numerical results and the BO solitons, as well as the experimental data, the internal waves propagating over the uniformly sloping beach are simulated including the cases of the mild and long slopes. The internal waves show remarkable shoaling after the interface touches the critical level. In the lower layer, the horizontal velocity becomes larger than the local linear celerity of internal waves in shallow water just before the crest peak and the position is defined as the wave-breaking point when the ratio of nonlinear parameter to beach slope is large. The ratio of initial wave height to wave-breaking depth becomes larger as the slope is milder and the wave nonlinearity is stronger. The wave height does not increase so much before the wave breaking on the mildest slope.
\end{abstract}

Keywords: nonlinear internal wave; two-layer system; variational principle; shoaling; wave-breaking point

\section{INTRODUCTION}

When density stratification is well developed in a nearshore zone, internal waves propagate affecting the water environment. Especially in shallow water regions, not only internal long-period waves, e.g. internal seiches and tides, but also internal short-period waves are observed with wave nonlinearity and dispersion. Internal waves propagating over a slope have been studied through hydraulic experiments (e.g. Helfrich, 1992), as well as various theories including the Benjamin-Ono (BO) equation considering both weak nonlinearity and weak dispersion of waves.

In the present study, a set of nonlinear internal wave equations derived on the basis of the variational principle without any assumptions on wave nonlinearity and dispersion (Kakinuma, 2001) is numerically solved in the vertical two-dimension. In the derivation process of equations, the velocity potential is expanded into a power series of vertical position, after which the velocity potential is approximated using only several terms of the power series in numerical computation. It has been confirmed that the numerical model shows good results in comparison with experimental data over a flat seabed (Yamashita et al., 2011) when the number of terms for the velocity potential is sufficient.

First, the numerical model of internal waves is verified: in deep-water cases, computational results of interface profiles up to each order on the vertical length scale of motion are compared with the theoretical solutions of the BO equation; on the other hand, in shallow-water cases, numerical calculation results obtained through the present model are compared with those through the fully nonlinear model for long internal waves (Choi and Camassa, 1999), as well as the existing experimental data (Horn et al., 2000).

Second, physical variables are evaluated as internal waves are approaching to wave-breaking points to investigate characteristics of nonlinear internal waves propagating on uniformly sloping beaches of mild and long slopes, which are difficult to be represented in a laboratory tank.

\section{NORMALIZED EQUATIONS FOR NONLINEAR INTERNAL WAVES}

In two-layer density stratification of inviscid and incompressible fluids between two fixed horizontal plates, the still-water thickness of the $i$-layer is denoted by $h_{i}(\boldsymbol{x})$. None of the fluids mix even in motion and the density $\rho_{i}\left(\rho_{1}<\rho_{2}\right)$ is spatially uniform and temporally constant in each layer. Surface tension and capillary action are neglected.

Fluid motion is assumed to be irrotational, such that the velocity potential $\phi_{i}$ is expanded into a series in terms of a given set of vertically distributed functions $Z_{i, \alpha}$ multiplied by their weightings $f_{i, \alpha}$ as

$$
\phi_{i}(\boldsymbol{x}, z, t)=\sum_{\alpha=0}^{N-1}\left\{Z_{i, \alpha}(z) \cdot f_{i, \alpha}(\boldsymbol{x}, t)\right\} \equiv Z_{i, \alpha} f_{i, \alpha},
$$

where the sum rule of product is adopted for subscript $\alpha$.

Nonlinear internal wave eqations based on the variational principle are as follows (Yamashita et al., 2011):

\footnotetext{
${ }^{1}$ Division of Natural Science, Graduate School of Science and Engineering, Kagoshima University, 1-21-40 Korimoto, Kagoshima, Kagoshima 890-0065, Japan

2 Ditto

${ }^{3}$ Department of Civil and Environmental Engineering, Kitami Institute of Technology, 165 Koen-cho, Kitami, Hokkaido 090-8507, Japan.
} 


$$
\begin{gathered}
\eta^{\alpha} \frac{\partial \eta}{\partial t}+\frac{1}{\alpha+\beta+1} \nabla\left(\eta^{\alpha+\beta+1} \nabla f_{1, \beta}\right)-\frac{\alpha \beta}{\alpha+\beta-1} \eta^{\alpha+\beta-1} f_{1, \beta}=0 \\
\eta^{\alpha} \frac{\partial \eta}{\partial t}+\frac{1}{\alpha+\beta+1} \nabla\left\{\left(\eta^{\alpha+\beta+1}-b^{\alpha+\beta+1}\right) \nabla f_{2, \beta}\right\}-\frac{\alpha \beta}{\alpha+\beta-1}\left(\eta^{\alpha+\beta-1}-b^{\alpha+\beta-1}\right) f_{2, \beta}=0, \\
\eta^{\beta} \frac{\partial f_{2, \beta}}{\partial t}+\frac{1}{2} \eta^{\beta+\gamma} \nabla f_{2, \beta} \nabla f_{2, \gamma}+\frac{1}{2} \beta \gamma \eta^{\beta+\gamma-2} f_{2, \beta} f_{2, \gamma}+g\left(\eta+h_{1}\right) \\
-\frac{\rho_{1}}{\rho_{2}}\left\{\eta^{\beta} \frac{\partial f_{1, \beta}}{\partial t}+\frac{1}{2} \eta^{\beta+\gamma} \nabla f_{1, \beta} \nabla f_{1, \gamma}+\frac{1}{2} \beta \gamma \eta^{\beta+\gamma-2} f_{1, \beta} f_{1, \gamma}+g\left(\eta+h_{1}\right)\right\}=0
\end{gathered}
$$

where $\nabla=(\partial / \partial x, \partial / \partial y)$, i.e., a partial differential operator in the horizontal plane; $\eta(\boldsymbol{x}, t), b(\boldsymbol{x})$, and $g$ are interface displacement, seabed position, and gravitational acceleration, respectively.

The physical variables are normalized as

$$
\begin{aligned}
& x^{*}=\frac{x}{\lambda}, y^{*}=\frac{y}{\lambda}, z^{*}=\frac{z}{\sum_{k=1}^{i} h_{k}}, t^{*}=\frac{\sqrt{g h}}{\lambda} t, \nabla^{*}=\lambda \nabla, \frac{\partial}{\partial t^{*}}=\frac{\lambda}{\sqrt{g h}} \frac{\partial}{\partial t}, \\
& \eta^{*}=\frac{\eta}{h_{1}}, b^{*}=\frac{b}{h}, f_{i, \alpha}^{*}=\frac{\left(\sum_{k=1}^{i} h_{k}\right)^{\alpha}}{\lambda \sqrt{g h}} \frac{h_{i}}{a} f_{i, \alpha}, \delta=\frac{h_{1}}{h}, \quad \varepsilon_{i}=\frac{a}{h_{i}}, \quad \sigma_{i}=\frac{h_{i}}{\lambda},
\end{aligned}
$$

where $h, \lambda$, and $a$ are characteristic water depth, wavelength, and wave height, respectively. These equations are substituted into Eqs. (2), (3), and (4), resulting in

$$
\begin{gathered}
\left(\eta^{*}\right)^{\alpha} \frac{\partial \eta^{*}}{\partial t^{*}}+\frac{1}{\alpha+\beta+1} \varepsilon_{1} \nabla^{*}\left(\left(\eta^{*}\right)^{\alpha+\beta+1} \nabla^{*} f_{1, \beta}^{*}\right\}-\frac{\alpha \beta}{\alpha+\beta-1} \frac{\varepsilon_{1}}{\sigma_{1}^{2}}\left(\eta^{*}\right)^{\alpha+\beta-1} f_{1, \beta}^{*}=0, \\
\eta^{*} \frac{\partial \eta^{*}}{\partial t^{*}}+\frac{1}{\alpha+\beta+1} \delta^{\beta} \varepsilon_{2} \nabla^{*}\left\{\left[\left(\eta^{*}\right)^{\alpha+\beta+1}-\left(\frac{b^{*}}{\delta}\right)^{\alpha+\beta+1}\right] \nabla^{*} f_{2, \beta}^{*}\right\} \\
-\frac{\alpha \beta}{\alpha+\beta-1} \frac{\delta^{\beta} \varepsilon_{2}}{\sigma_{1}^{2}}\left\{\left(\eta^{*}\right)^{\alpha+\beta-1}-\left(\frac{b^{*}}{\delta}\right)^{\alpha+\beta-1}\right\} f_{2, \beta}^{*}=0, \\
\delta^{\beta} \varepsilon_{2}\left(\eta^{*}\right)^{\beta} \frac{\partial f_{2, \beta}^{*}}{\partial t^{*}}+\frac{1}{2} \delta^{\beta+\gamma} \varepsilon_{2}^{2}\left(\eta^{*}\right)^{\beta+\gamma} \nabla^{*} f_{2, \beta}^{*} \nabla^{*} f_{2, \gamma}^{*} \\
+\frac{1}{2} \beta \gamma \delta^{\beta+\gamma}\left(\frac{\varepsilon_{2}}{\sigma_{1}}\right)^{2}\left(\eta^{*}\right)^{\beta+\gamma-2} f_{2, \beta}^{*} f_{2, \gamma}^{*}+\delta\left(\eta^{*}+1\right) \\
-\frac{\rho_{1}}{\rho_{2}}\left\{\varepsilon_{1}\left(\eta^{*}\right)^{\beta} \frac{\partial f_{1, \beta}^{*}}{\partial t^{*}}+\frac{1}{2} \varepsilon_{1}^{2}\left(\eta^{*}\right)^{\beta+\gamma} \nabla^{*} f_{1, \beta}^{*} \nabla^{*} f_{1, \gamma}^{*}\right. \\
\left.+\frac{1}{2} \beta \gamma\left(\frac{\varepsilon_{1}}{\sigma_{1}}\right)^{2}\left(\eta^{*}\right)^{\beta+\gamma-2} f_{1, \beta}^{*} f_{1, \gamma}^{*}+\delta\left(\eta^{*}+1\right)\right\}=0 .
\end{gathered}
$$

\section{NUMERICAL CALCULATION METHOD}

Two-layer problems are solved in vertically two-dimensional cases. Equations (6), (7), and (8) are rewritten to finite difference equations, after which the time development is carried out by applying implicit schemes similar to that of Nakayama and Kakinuma (2010).

\section{COMPARISON BETWEEN NUMERICAL RESULTS AND THEORETICAL SOLUTIONS}

\section{Deep-Water Cases}

We compare computational results of interface profiles with theoretical solutions through the BO equation. In the derivation process of the BO equation, the depth of one layer is assumed to be inifinitely deep. In this study, it is assumed that the lower layer thickness is much larger than that of the upper layer as shown in Fig. 1 and $a / h_{1}=O\left(h_{1} / \lambda\right)^{2}<<1$. The BO equation (Benjamin, 1966; Ono, $1975)$ is 


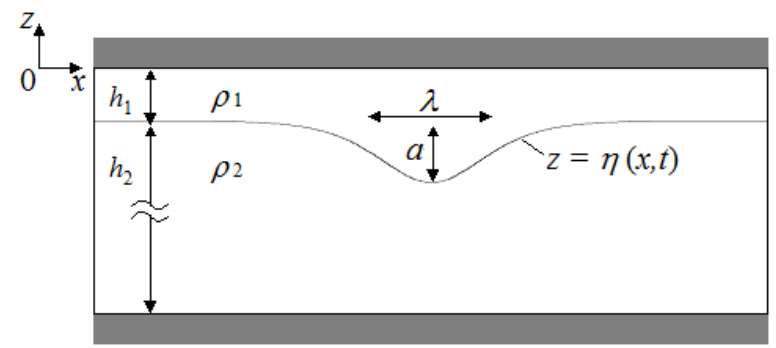

Figure 1. Two-layer system between two fixed horizontal plates.

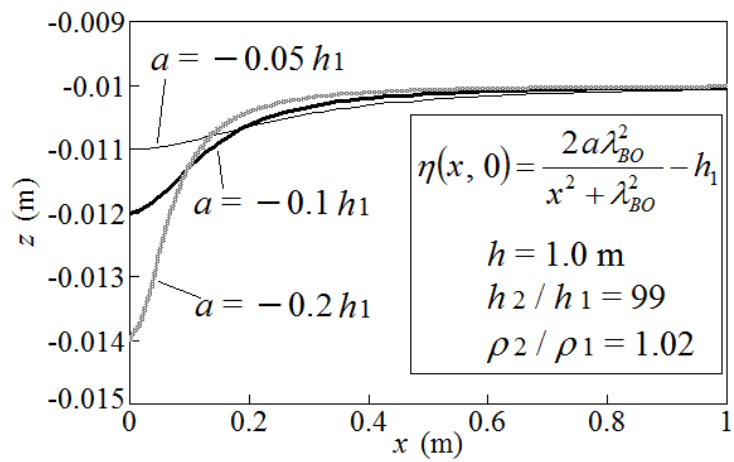

Figure 2. Initial interface profiles (The initial wave height is equal to 2a.).

$$
\frac{\partial \eta}{\partial t}+c_{0} \frac{\partial \eta}{\partial x}+c_{1} \eta \frac{\partial \eta}{\partial x}+c_{2} H\left(\frac{\partial^{2} \eta}{\partial x^{2}}\right)=0
$$

where $H\left(\partial^{2} \eta / \partial x^{2}\right)$ is Hilbert transform of $\partial^{2} \eta / \partial x^{2}$. The theoretical solution of a solitary wave obtained by the $\mathrm{BO}$ equation, i.e., a BO soliton, is

$$
\begin{gathered}
\eta(x, t)=\frac{a_{0} \lambda_{\mathrm{BO}}^{2}}{(x-c t)^{2}+\lambda_{\mathrm{BO}}^{2}}-h_{1}, \\
c=c_{0}+\frac{1}{4} a_{0} c_{1}, \lambda_{\mathrm{BO}}=\frac{4 c_{2}}{a_{0} c_{1}}, c_{0}=\sqrt{\frac{\rho_{2}-\rho_{1}}{\rho_{1}} g h_{1}}, c_{1}=-\frac{3 c_{0}}{2 h_{1}}, c_{2}=\frac{c_{0} h_{1}}{2} \frac{\rho_{2}}{\rho_{1}} .
\end{gathered}
$$

In numerical computation, the initial interface profiles are shown in Fig. 2, where the interface displacement $\eta(x, 0)=2 a \lambda_{\mathrm{BO}}{ }^{2} /\left(x^{2}+\lambda_{\mathrm{BO}}{ }^{2}\right)-h_{1} ; \lambda_{\mathrm{BO}}$ is the characteristic wavelength of $\mathrm{BO}$ soliton; the initial total depth $h$, thickness ratio $h_{2} / h_{1}$, and density ratio $\rho_{2} / \rho_{1}$ are $1.0 \mathrm{~m}, 99.0$, and 1.02 , respectively. There is a vertical wall of perfect reflection at $x=0 \mathrm{~m}$. The grid width $\Delta x$ and the time-step interval $\Delta t$ are equal to $0.02 \mathrm{~m}$ and $0.005 \mathrm{~s}$, respectively.

Time variation of the ratio of wave height to upper layer thickness, $\varepsilon_{1}^{\prime}$, and the ratio of upper layer thickness to wavelength, $\sigma_{1}^{\prime}$, are shown in Figs. 3(a) and 3(b), where the initial wave height is equal to $0.05 h_{1},-0.1 h_{1}$, and $-0.2 h_{1} ; C_{0}$ is celerity of linear internal wave in shallow water; the ratios $\varepsilon_{1}^{\prime}$ and $\sigma_{1}^{\prime}$ are defined by Koop and Butler (1981) as

$$
\varepsilon_{1}^{\prime}=\frac{\left|\eta_{\text {trough }}+h_{1}\right|}{h_{1}}, \sigma_{1}^{\prime}=\frac{h_{1}}{\frac{2}{\varepsilon_{1}^{\prime} h_{1}} \int_{x_{\text {trough }}}^{+\infty}\left(\eta+h_{1}\right) d x},
$$

where $\eta_{\text {trough }}$ and $x_{\text {trough }}$ are interface displacement and horizontal position of the first trough of internal wave, respectively.

If $a=-0.05 h_{1}$ and $N=3,4$, or 5 , then $\varepsilon_{1}^{\prime}$ and $\sigma_{1}^{\prime}$ have become steady when $t C_{0} / h_{1}>2,000$; on the other hand, if $a=-0.2 h_{1}$ and $N=4$ or 5 , then $\varepsilon_{1}^{\prime}$ and $\sigma_{1}^{\prime}$ have become relatively steady when $t C_{0} / h_{1}>$ 


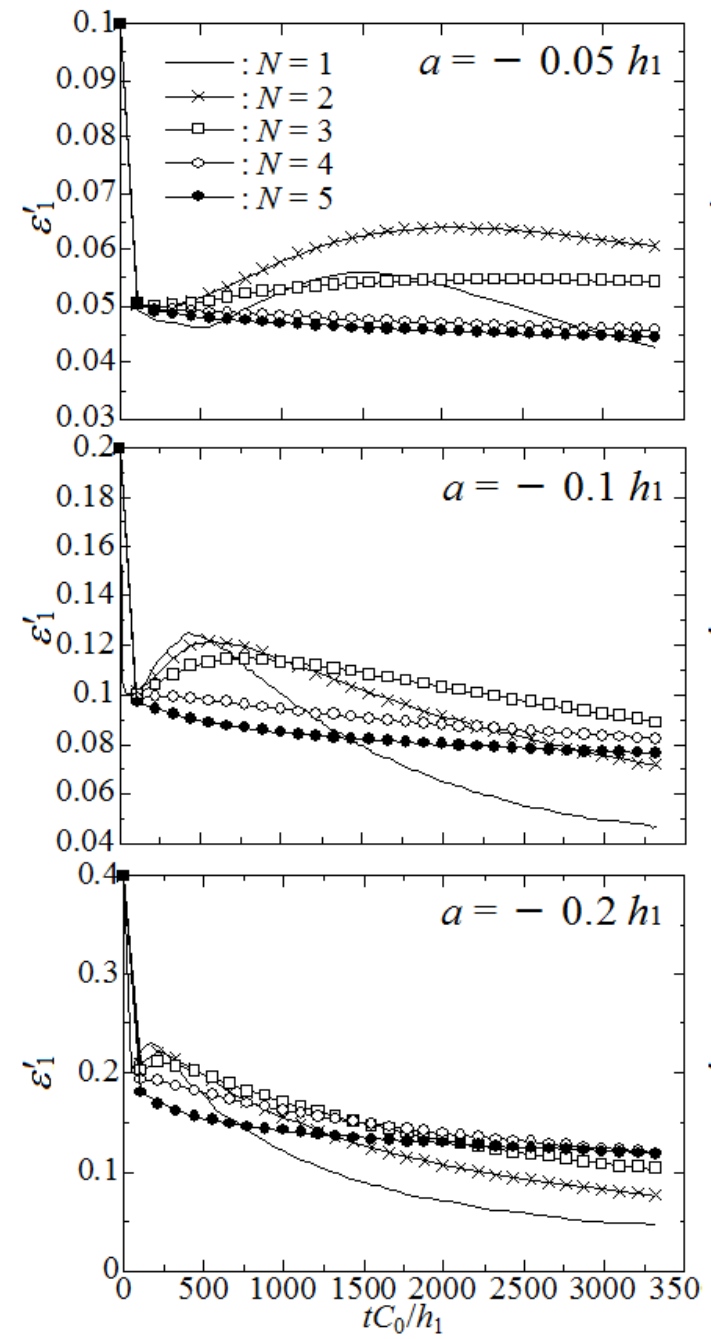

(a)
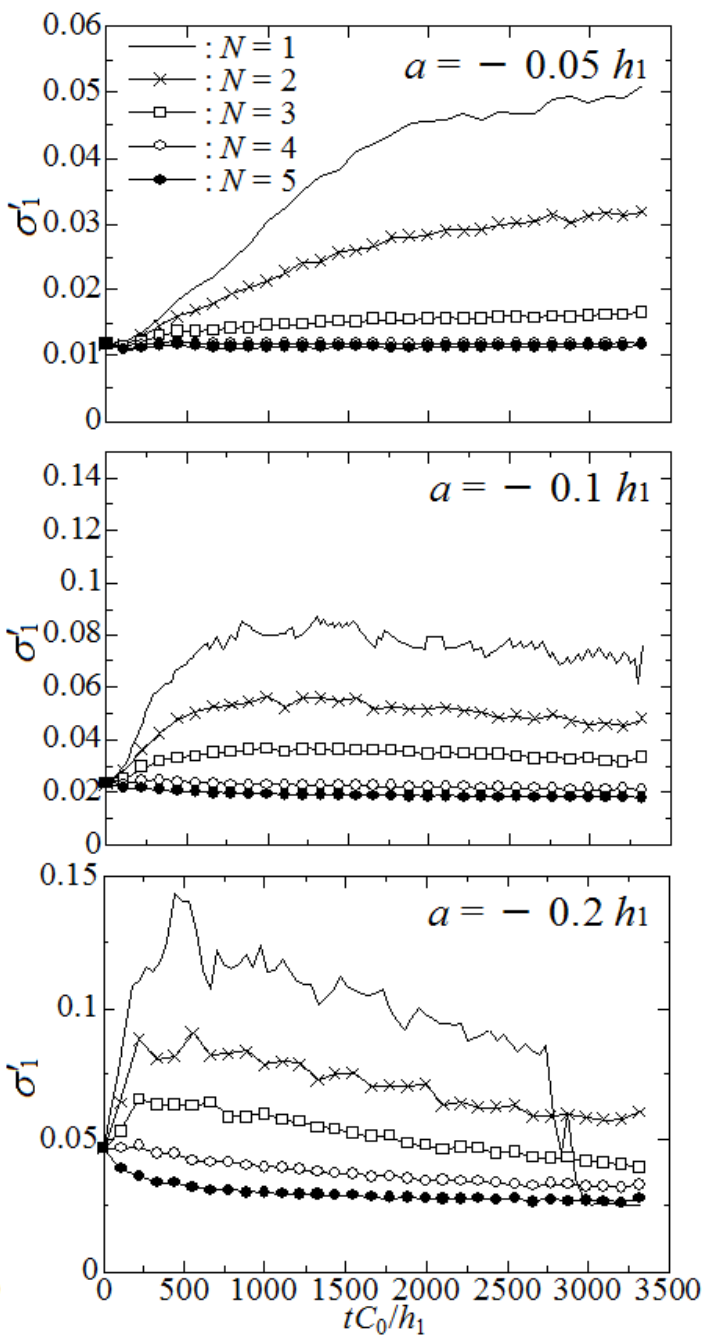

(b)

Figure 3. Time variation of the ratio of wave height to upper layer thickness (a) and the ratio of upper layer thickness to wavelength (b), where $C_{0}$ is the celerity of linear internal wave in shallow water.

3,000. In the verification of the model, the stationary waves should be compared with BO solitons.

In Fig. 4, numerical results of interface profiles at $t C_{0} / h_{1}=3,320$ are compared with those of the corresponding BO solitons, where $a=-0.05 h_{1}$ and $a_{0}=\eta_{\text {trough }}+h_{1}$. When $N<4$, the interface gradient is steeper than that of the BO soliton. On the other hand, when $N=4$ or 5 , the interface profile obtained through the present model is in harmony with that of the $\mathrm{BO}$ soliton. When $N=5, \varepsilon_{1}^{\prime}, \sigma_{1}^{\prime}$, and the ratio of lower layer thickness to wavelength, $\sigma_{2}^{\prime}$, are equal to $0.045,0.01$, and 0.99 , respectively, at $t C_{0} / h_{1}=$ 3,320 . The result through the linearized present model (linear model) is remarkably disintegrated due to the wave dispersion.

Figures 5(a) and 5(b) show vertical distributions of horizontal velocity in the upper layer above the

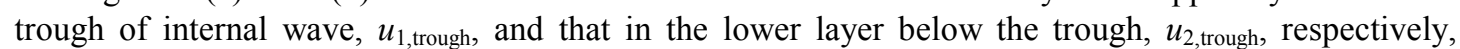
where $a=-0.05 h_{1}$ and $t C_{0} / h_{1}=3,320$. The distributions of $u_{1 \text {,trough }}$ are nearly uniform since the wavelength is much larger than the upper layer thickness and the wave nonlinearity is not so strong.

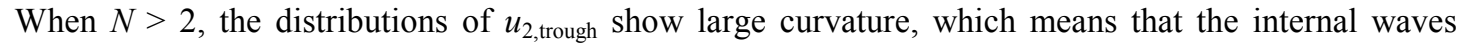
propagate in rather deep water.

Calculation results of interface profiles at $t C_{0} / h_{1}=3,320$ are compared with those of the corresponding BO solitons in Fig. 6 , where $a=-0.2 h_{1}$. When $N=5, \varepsilon_{1}^{\prime}, \sigma_{1}^{\prime}$, and $\sigma_{2}^{\prime}$ are equal to 0.12 , 0.03 , and 2.97, respectively, at $t C_{0} / h_{1}=3,320$. In this case, where the wave nonlinerity is stronger $\left(a / h_{1}\right.$ $=0.2$ ), the wave profiles obtained through the present model are similar to those of the BO solitons 

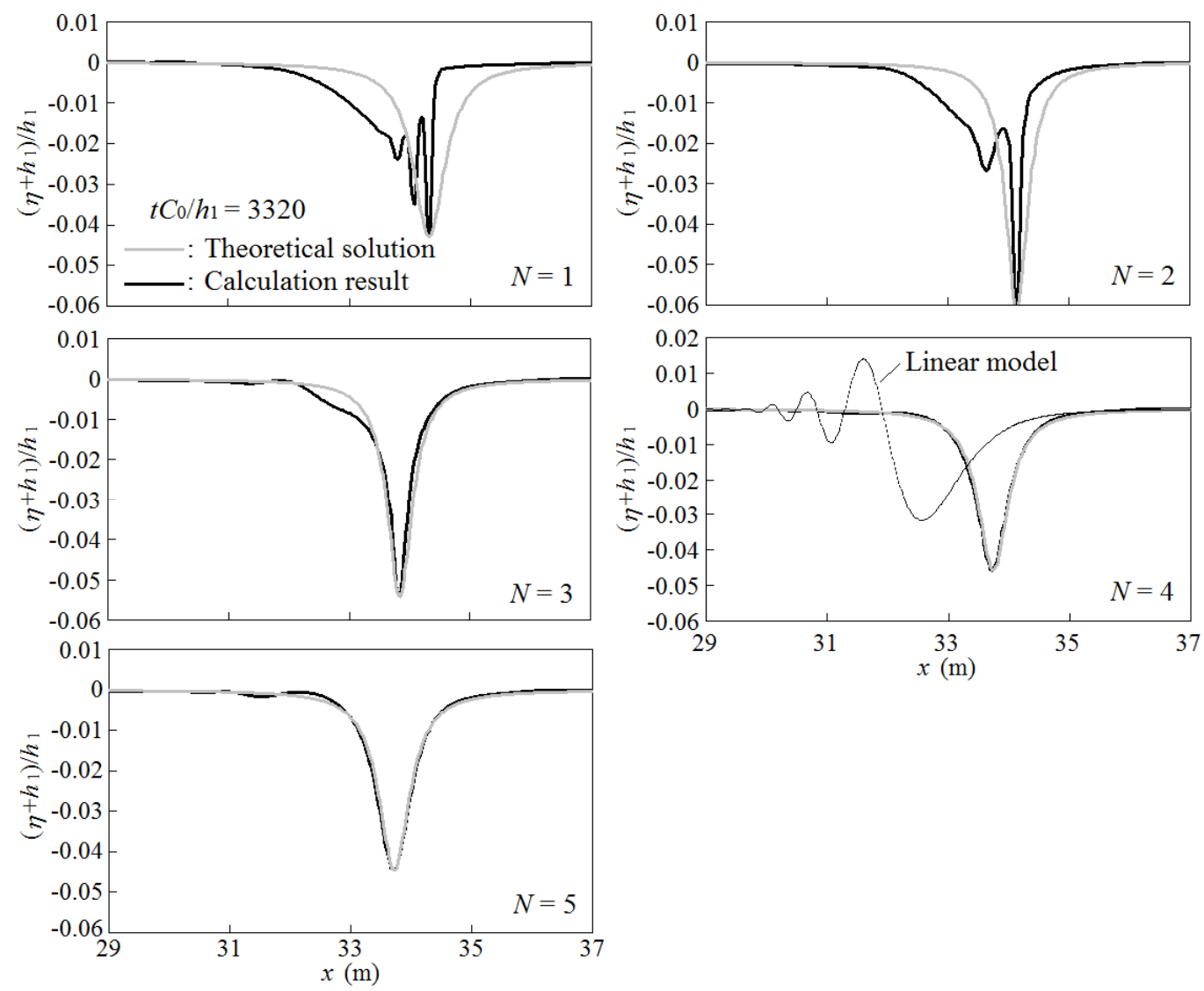

Figure 4. Interface profiles at $t C_{0} / h_{1}=3,320$, where $h_{2} / h_{1}=99.0$ and $a=-0.05 h_{1}$.

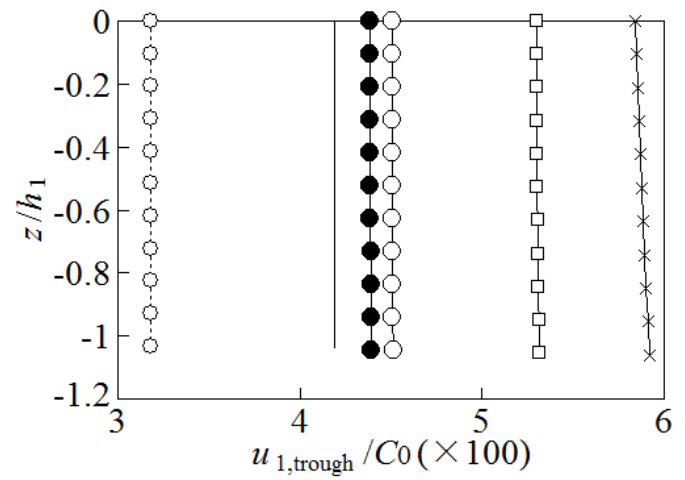

(a)

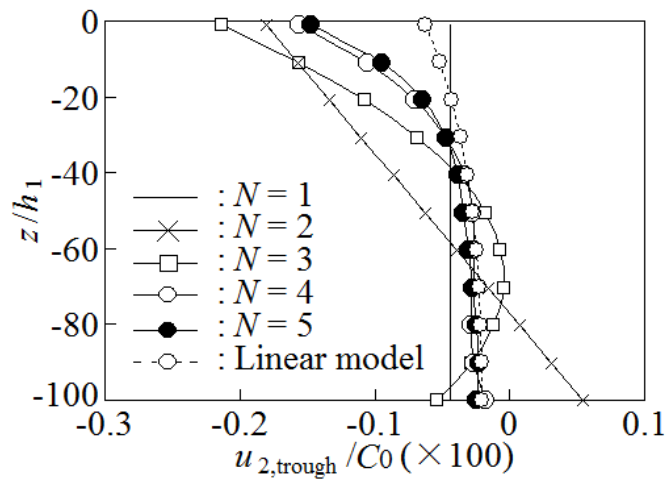

(b)

Figure 5. Vertical distributions of horizontal velocity in the upper layer above the trough of internal wave, $u_{1, \text { trough, }}$ and that in the lower layer below the trough of internal wave, $u_{2}$,trough.

when $N>3$. However, the interface gradient through the present model is milder than that of the $\mathrm{BO}$ soliton as shown in Fig. 7, which means that the present model considers wave nonlinearity better than the BO equation since the interface gradient of strongly nonlinear wave is milder than that through the theories for weakly nonlinear waves as the KdV theory (Nakayama and Kakinuma, 2010).

Figure 8 shows the numerical calculation results of wave profiles at $t C_{0} / h_{1}=3,320$ in comparison with those of the corresponding BO solitons in a deeper case, where $h=10.0 \mathrm{~m}, h_{2} / h_{1}=999.0$, and the density ratio $\rho_{2} / \rho_{1}$ is 1.02 ; the initial interface displacement $\eta(x, 0)=2 a \lambda_{\mathrm{BO}}{ }^{2} /\left(x^{2}+\lambda_{\mathrm{BO}}{ }^{2}\right)-h_{1}$ and $a=$ $-0.05 h_{1}$; the grid width $\Delta x$ and the time-step interval $\Delta t$ are equal to $0.02 \mathrm{~m}$ and $0.005 \mathrm{~s}$, respectively. 


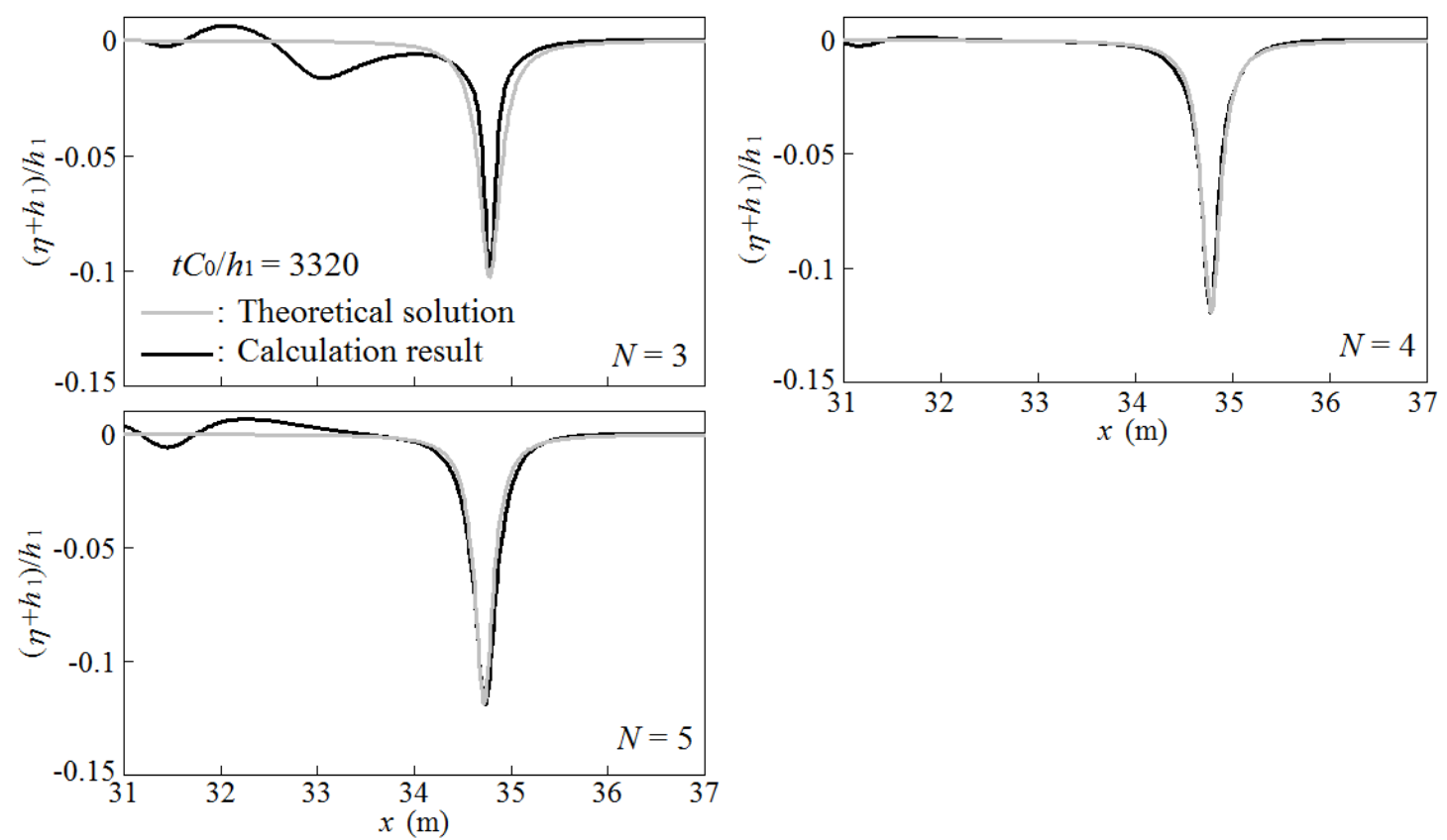

Figure 6. Interface profiles at $t C_{0} / h_{1}=3,320$ where $h_{2} / h_{1}=99.0$ and $a=-0.2 h_{1}$.
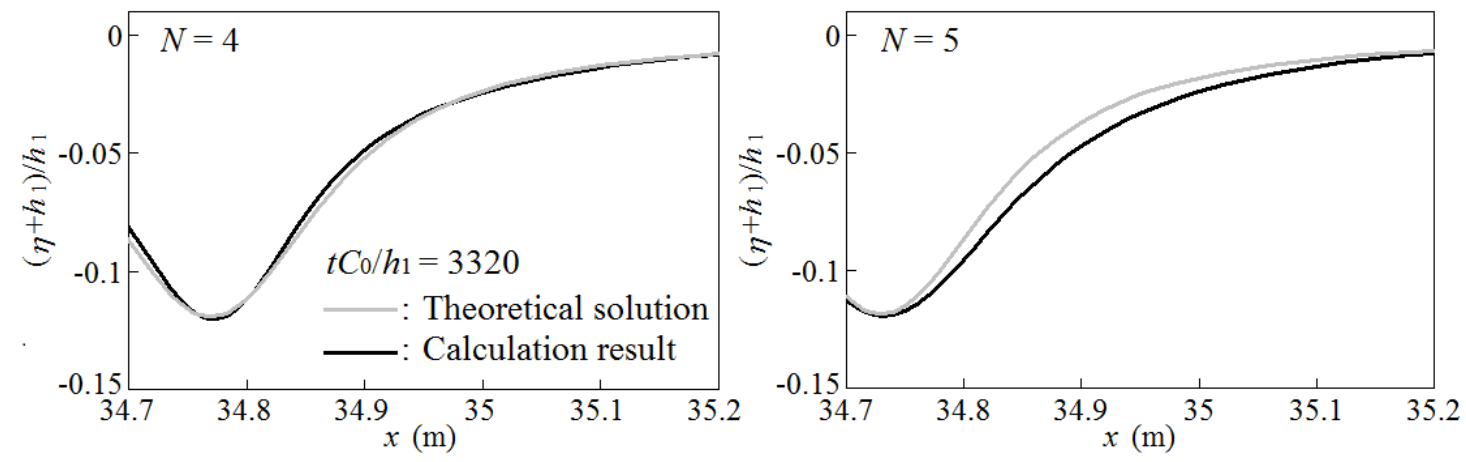

Figure 7. Interface profiles at $t C_{0} / h_{1}=3,320$, where $h_{2} / h_{1}=99.0$ and $a=-0.2 h_{1}$.
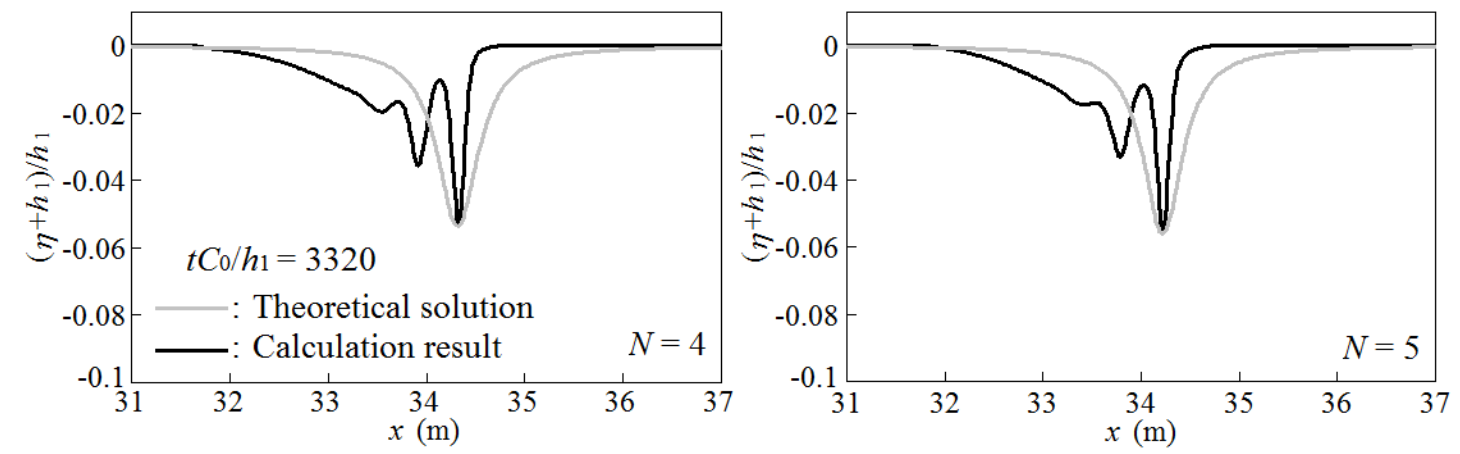

Figure 8. Interface profiles at $t C_{0} / h_{1}=3,320$, where $h_{2} / h_{1}=999.0$ and $a=-0.05 h_{1}$. 


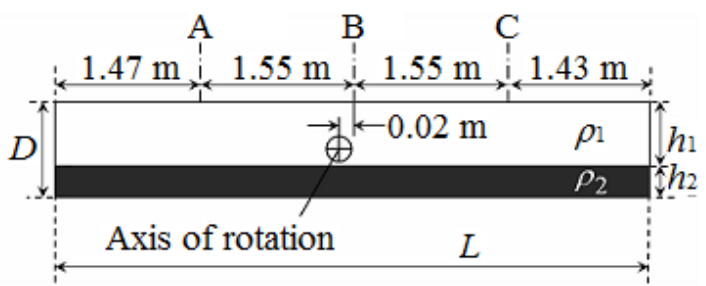

(a) Dimensions of the laboratory tank.

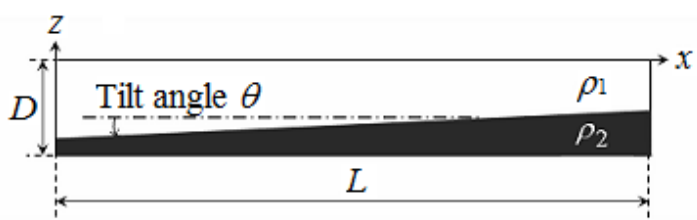

(b) Initial condition, where the interface is inclined linearly with the angle $\theta$ in the horizontal tank.

Figure 9. Schematic of tanks.
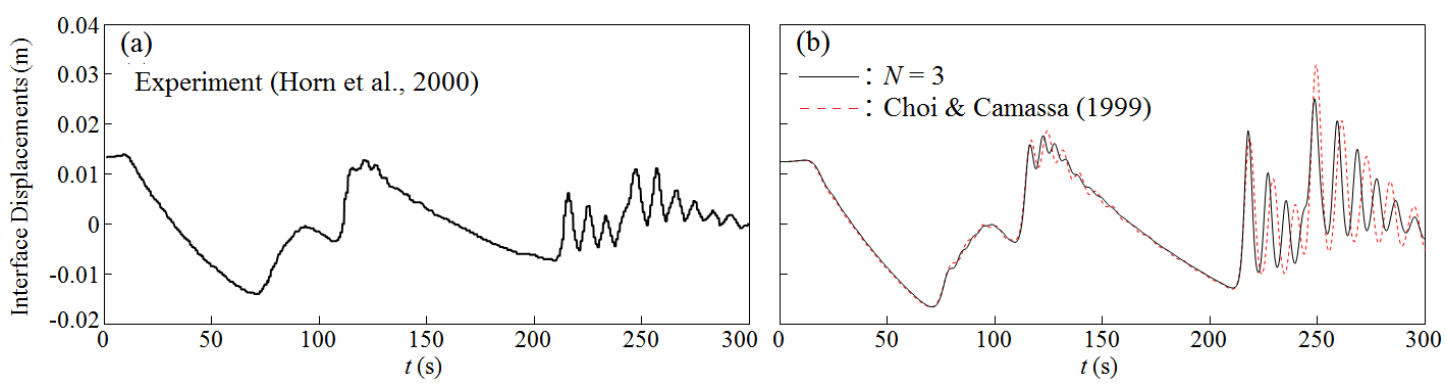

Figure 10. Time variation of interface displacement measured at Position $C$ in the hydraulic experiment and those through the present model, where the number of terms for expanded velocity potential, $N$, is equal to three, and the fully nonlinear model by Choi and Camassa (1999).

The profiles of $\mathrm{BO}$ solitons are much different from the calculation results, where larger number of terms for the velocity potential are required to represent the wave dispersion more accurately.

\section{Shallow-Water Case}

Horn et al. (2000) performed hydraulic experiments using a tank, where the length $L$, depth $D$, and width $W$ were $6.0,0.29$, and $0.3 \mathrm{~m}$, respectively, as shown in Fig. 9(a). Three ultra-sonic wave gauges were set at the Positions A, B, and C. The tank was filled with a two-layer stratification, where $h_{1} / D=$ 0.8 . At the beginning of experiments, the tilted tank, where the tilt angle was $\theta$ around the axis of rotation, was returned to a horizontal position quickly, after which internal waves traveled in the twolayer system between two fixed horizontal plates.

In the initial condition of numerical computation, the tank is horizontal and the interface is inclined linearly as shown in Fig. 9(b); the initial velocity potential is assumed to be zero through the computational domain. The grid width $\Delta x$ and the time-step interval $\Delta t$ are equal to $0.06 \mathrm{~m}$ and $0.02 \mathrm{~s}$, respectively.

The interface displacement at Position C in Fig. 9(a) measured in the experiment is shown in Fig. 10 (a), while the corresponding calculation result through the present model, where $N=3$, and the fully nonlinear model for long internal waves by Choi and Camassa (1999) (the CC model), where $O\left(\varepsilon_{1}\right)=1$ and $O\left(\sigma_{1}\right)^{4}<<1$, are shown in Fig. 10(b), in the case where the density ratio $\rho_{2} / \rho_{1}$ and tilt angle $\theta$ are 1.019 and $0.4617^{\circ}$, respectively. The calculation result through the present model represents the accurate wave periods, although the wave height through the numerical models is too large because the numerical computation does not consider viscosity of the fluids and friction at the interface. When $t>$ $240 \mathrm{~s}$, the number of crests through both the experiment and the present model is equal to six, whereas that through the $\mathrm{CC}$ model is five.

The interface profiles at $t=280 \mathrm{~s}$ obtained using the present model, where $N=3$ or 4 , the CC model, and a Boussinesq-type model, where $O\left(\varepsilon_{1}\right)=O\left(\sigma_{1}\right)^{2}<<1$, are shown in Fig. 11. The wave height through the present and the CC models is larger than that trough the Boussinesq-type weakly nonlinear model. On the other hand, the wavelength through the present model is shorter than that through the CC model and the Boussinesq-type weak dispersion model. 


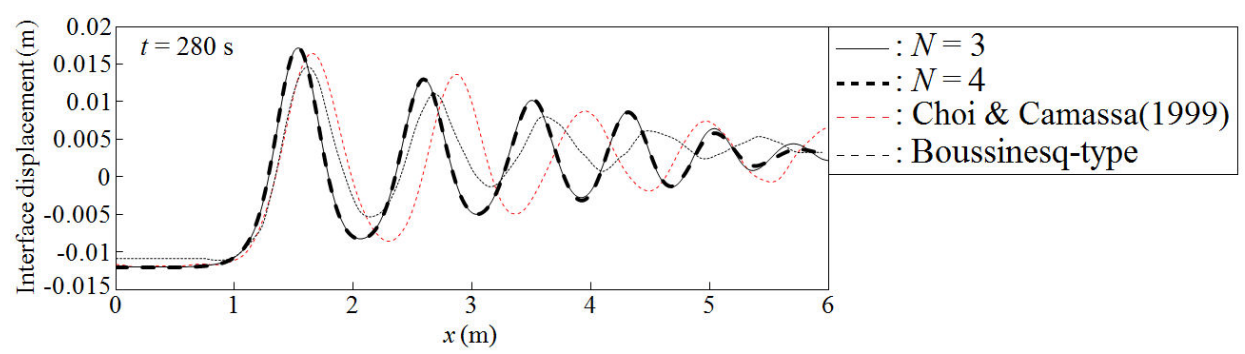

Figure 11. Interface profiles through the present model, where $\boldsymbol{N}=\mathbf{3}$ and 4 , the fully nonlinear model by Choi and Camassa (1999), and the Boussinesq-type model at $t=280 \mathrm{~s}$.

\begin{tabular}{|c|c|c|c|}
\hline$\rho_{2} / \rho_{1}$ & $h_{1} / h$ & $a_{0} / h_{1}$ & $s=\tan \theta$ \\
\hline \multirow[t]{5}{*}{1.02} & \multirow[t]{2}{*}{0.2} & 0.2 & $0.01,0.02,0.03,0.04,0.05,0.06,0.07$ \\
\hline & & 0.3 & $0.01,0.02,0.03,0.04,0.05,0.06,0.07$ \\
\hline & \multirow[t]{2}{*}{0.3} & 0.2 & $0.01,0.02,0.03,0.04,0.05,0.06,0.07$ \\
\hline & & 0.3 & $0.01,0.02,0.03,0.04,0.05,0.06,0.07$ \\
\hline & 0.4 & 0.2 & $0.01,0.02,0.03,0.04,0.05,0.06,0.07$ \\
\hline \multirow[t]{5}{*}{1.024} & \multirow[t]{5}{*}{0.278} & 0.20 & 0.034 \\
\hline & & 0.21 & 0.050 \\
\hline & & 0.27 & $0.050, \quad 0.067$ \\
\hline & & 0.33 & 0.034 \\
\hline & & 0.34 & $0.050, \quad 0.067$ \\
\hline
\end{tabular}

Case A: $\rho_{2} / \rho_{1},=1.02, h_{1} / h,=0.2, a_{0} / h_{1}=0.2$, and $s=0.03$

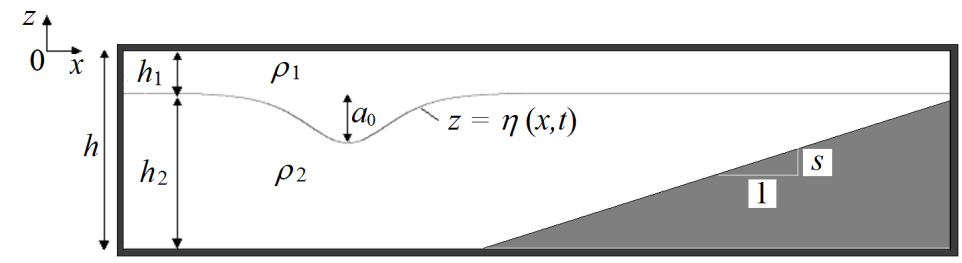

Figure 12. Initial interface profile over a uniformly sloping beach.

\section{NUMERICAL SIMULATION OF INTERNAL WAVES OVER A UNIFORMLY SLOPING BEACH}

\section{Definition of Wave-Breaking Point}

Helfrich (1992) performed hydraulic experiments on both breaking and run-up of internal solitary waves on a uniform slope using a water basin, where the beach slope $s$ was larger than 0.03 . In actual cases, the beach slope is, for example, equal to 0.01 in a lake or 0.001 on the sea shore. In the present paper, numerical simulation of internal solitary waves propagating on a uniformly sloping beach is conducted for the cases shown in Tab. 1 including cases of mild and long slopes, which are difficult to be represented in a laboratory experiment. The calculation domain is shown in Fig. 12.

The case where $\rho_{2} / \rho_{1}, h_{1} / h, a_{0} / h_{1}$, and $s$ are $1.02,0.2,0.2$, and 0.03 , respectively, is reffered to as Case A. The incident waves are the third order theoretical solitary waves. The conditions where the density ratio $\rho_{2} / \rho_{1}=1.024$ correspond to the experimental conditions by Helfrich (1992). The lateral boundary condition is the perfect reflection at a vertical wall. The numerical calculation stops when the interface touches the bottom. The number of terms in expanded velocity potential, $N$, is equal to three. The grid width $\Delta x$ and the time-step interval $\Delta t$ are equal to $0.05 \mathrm{~m}$ and $0.005 \mathrm{~s}$, respectively.

Numerical results of interface profiles in Case A is shown in Fig. 13(a), where the dashed line shows the critical level, i.e., the lowest position for an interface of internal solitary wave to be able to appear. The critical level $z_{\mathrm{c}}$ is determined by the $\mathrm{KdV}$ theory as 

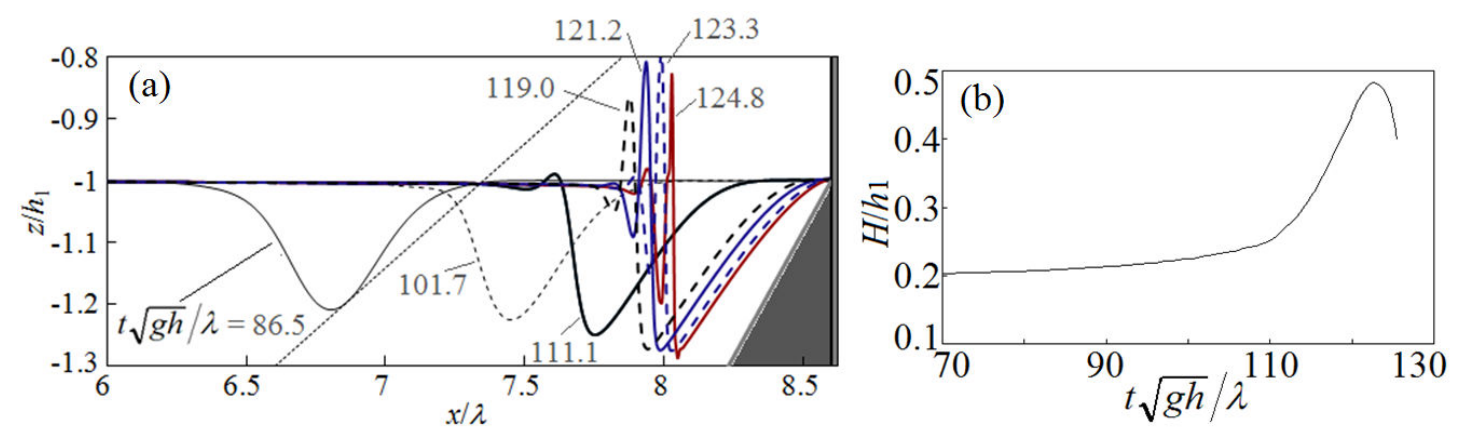

Figure 13. Time variation of interface profiles, where the dashed line shows the critical level obtained through the KdV theory, and the time variation of the ratio of wave height $H$ to upper layer thickness $h_{1}\left(\rho_{2} / \rho_{1}\right.$ $=1.02, h_{1} / h=0.2, a_{0} / h_{1}=0.2, s=0.03$ ).

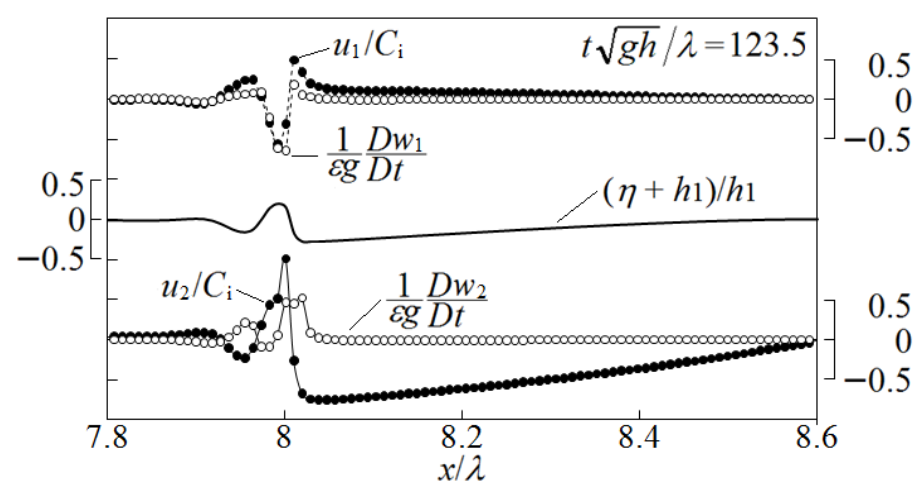

Figure 14. Interface profile, horizontal velocity of water particles in the vicinity of the interface in the $i$-layer, $u_{i}$, and vertical acceleration of water particles in the vicinity of the interface in the $i$-layer, $D w_{i} / D t$, where $C_{\mathrm{i}}$ is the local celerity of linear internal wave in shallow water $\left(\rho_{2} / \rho_{1}=1.02, h_{1} / h=0.2, a_{0} / h_{1}=0.2, s=0.03\right)$.

$$
z_{\mathrm{c}}=b /\left(1+\sqrt{\rho_{2} / \rho_{1}}\right)
$$

According to Fig. 13(a), when the interfaces reach the critical level, the internal waves propagating on the slope begin inclined backward, after which the internal waves show remarkable disintegration.

Time variation of the ratio of wave height to upper layer thickness in Case A is shown in Fig. 13(b), where the wave height $H$ is the vertical distance between the interface levels at the first trough and the first crest. The ratio increases gradually to have a peak at $t^{*}=t \sqrt{g h} / \lambda \approx 123 \mathrm{~s}$, after which it decreases.

The physical variables at $t^{*}=123.5 \mathrm{~s}$, including the interface profile, the horizontal velocities of water particles in the vicinity of the interface in the $i$-layer, $u_{i}$, and the vertical acceleration of water particles in the vicinity of the interface in the $i$-layer, $D w_{i} / D t$, are shown in Fig. 14, where $C_{\mathrm{i}}$ and $\varepsilon$ are local celerity of linear internal wave in shallow water and $\left(\rho_{2}-\rho_{1}\right) / \rho_{2}$, respectively. According to the figure, $u_{2}$ exceeds $C_{\mathrm{i}}$ just before the crest peak, where the position can be determined as the wavebreaking point. If $D w_{2} / D t$ becomes larger than $\varepsilon g$ before $u_{2}$ exceeds $C_{\mathrm{i}}$, where the position where $D w_{2} / D t$ becomes larger than $\varepsilon g$ is defined as the wave-breaking point. On the other hand, in the cases where $\gamma / s<3.5$, the interface touches the bottom before the internal waves break; these cases are plotted and indicated as 'No breaking' in Fig. 15, where the internal-wave-breaking points are summarized using a nonlinear parameter proposed by Aghsaee et al. (2010):

$$
\gamma=1.5 a\left|h_{1}-h_{2}\right| / h_{1} h_{2} .
$$

The amplitude of $\gamma$ indicates the strength of the wave nonlinearity. According to Fig. 15, internal waves break before the interface touches the bottom when $\gamma / s>3.5$, while internal waves break after the 


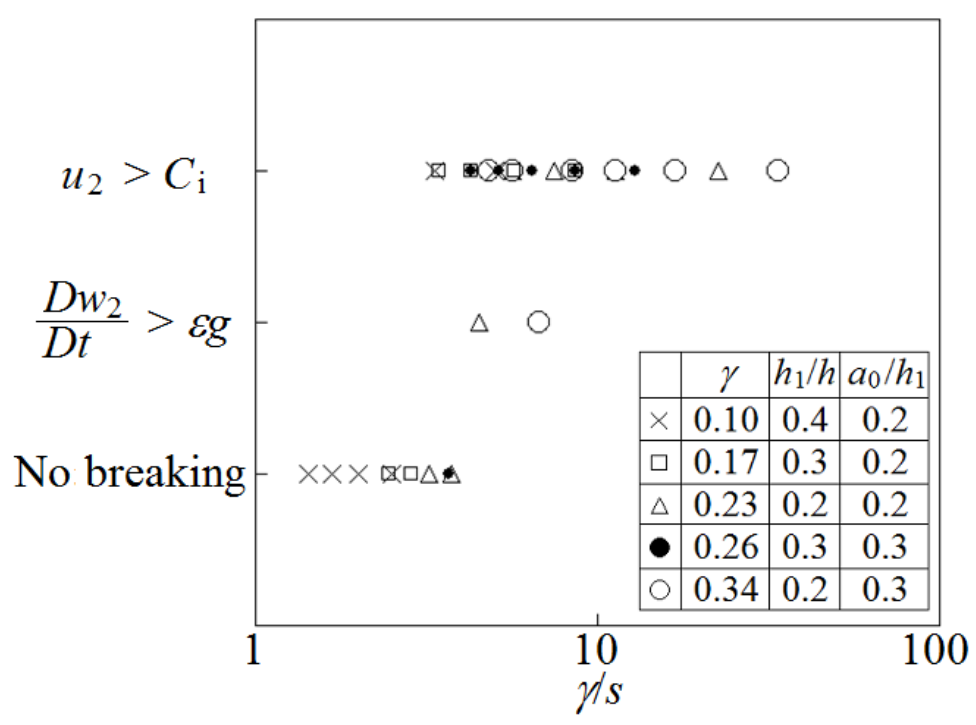

Figure 15. Conditions of internal-wave breaking.

interface touches the bottom when $\gamma / s<3.5$.

\section{Physical Variables near a Wave-Breaking Point}

Figure 16 shows the relationship between the raio of initial wave height $a_{0}$ to wave-breaking depth in the lower layer, $h_{2, \mathrm{BP}}$, and the ratio of nonlinear parameter $\gamma$ to slope $s$, where the suffix BP indicates the variable at a wave-breaking point. The calculation results are compared with the experimental data by Helfrich (1992) in Fig. 16, according to which the ratio $a_{0} / h_{2, \mathrm{BP}}$ is underestimated using the present model, which means that the wave-breaking depth in the lower layer, $h_{2, \mathrm{BP}}$, is overestimated. In the computation the friction is not considered at the interface and the incident waves are assumed to be the third order theoretical solitary waves, which may leads to such difference between the calculated and expermental results. The tendency of the data is, however, similar, where the larger the ratio $\gamma / s$ is, the larger the ratio $a_{0} / h_{2, \mathrm{BP}}$ becomes.

The relationship between $\gamma / s$ and $a_{0} / h_{2, \mathrm{BP}}$ is shown in Fig. 17, where the numerical results compensate the experimental data obtained by Helfirich (1992) in consideration of internal waves over the milder and longer slopes. Accoding to the figure, $a_{0} / h_{2, \mathrm{BP}}$ becomes larger as the slope is smaller and the wave nonlinearity is stronger.

The relation between the slope and the ratio of wave height $H_{\mathrm{BP}}$ to wave-breaking depth $h_{2, \mathrm{BP}}$ is shown in Fig. 18, where $H_{\mathrm{BP}}$ is the vertical distance of interface levels between at the first trough and the first crest, at the wave-breaking point. The value $H_{\mathrm{BP}} / h_{2, \mathrm{BP}}$ becomes larger, i.e., $0.9-1.8$, when the slope is milder, i.e., $s=0.03-0.01$.

Figure 19 shows the amplification factor of the wave height at the wave-breaking point, $H_{\mathrm{BP}} / a_{0}$. On the steeper slopes, internal waves show wave breaking before the wave height is amplified. On the mildest slope, the wave height does not increase so much in the wave shoaling because of the wave dispersion through the longer distance travel, where the energy of internal waves has been provided to the waves in the following wave train as shown in Fig. 20, where $\rho_{2} / \rho_{1}, h_{1} / h, a_{0} / h_{1}$, and $s$ are $1.02,0.2$, 0.3 , and 0.01 , respectively. On the other hand, the wave height is amplified remarkably when $s$ is equal to 0.02 to 0.04 .

The minimum interface gradient of the rear face of the first trough at the wave-breaking point, $\partial \eta_{\mathrm{BP}} /\left.\partial x\right|_{\min }$, is shown in Fig. 21. On the mildest slope where $s=0.01$, the rear face of the first trough at the wave-breaking point is milder than that of internal waves propagating on the steeper slopes.

\section{CONCLUSIONS}

The internal waves in the two-layer systems were numerically simulated by solving the set of nonlinear equations in consideration of both strong nonlinearity and strong dispersion of waves. After the verification of the numerical results in comparison with the BO solitons, as well as the existing experimental data, the internal waves propagating over the uniformly sloping beach were simulated including the cases of the mild and long slopes, which were difficult to be represented in a laboratory 


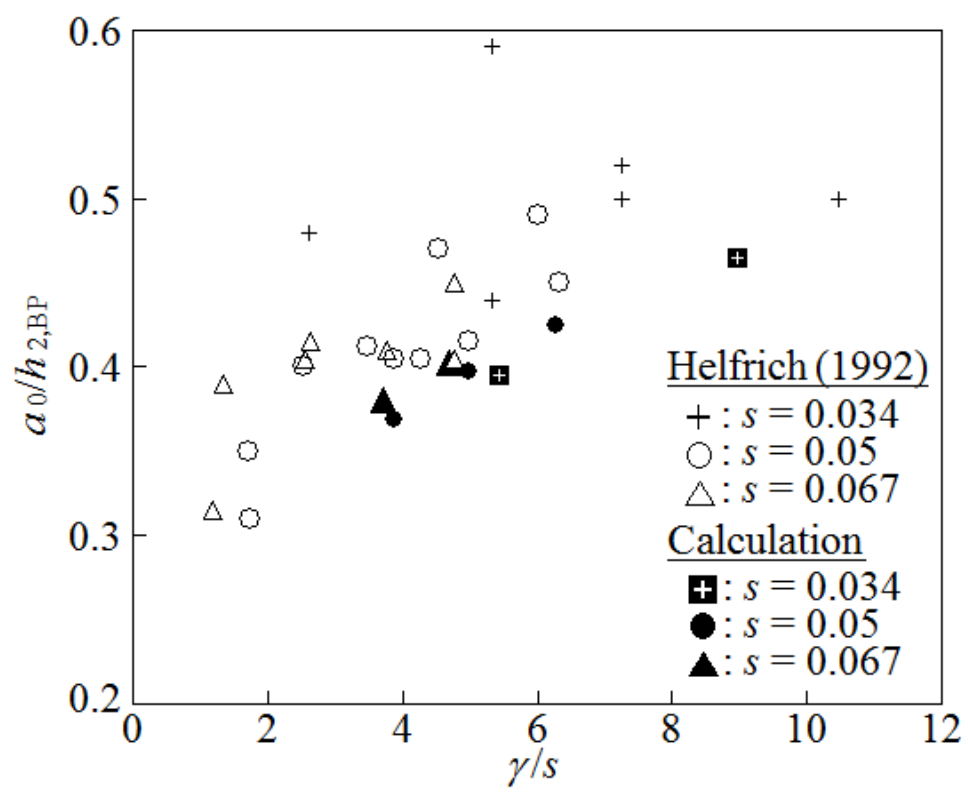

Figure 16. Location of wave-braking points through the present model, where $N=3$, and the experiments by Helfrich (1992).

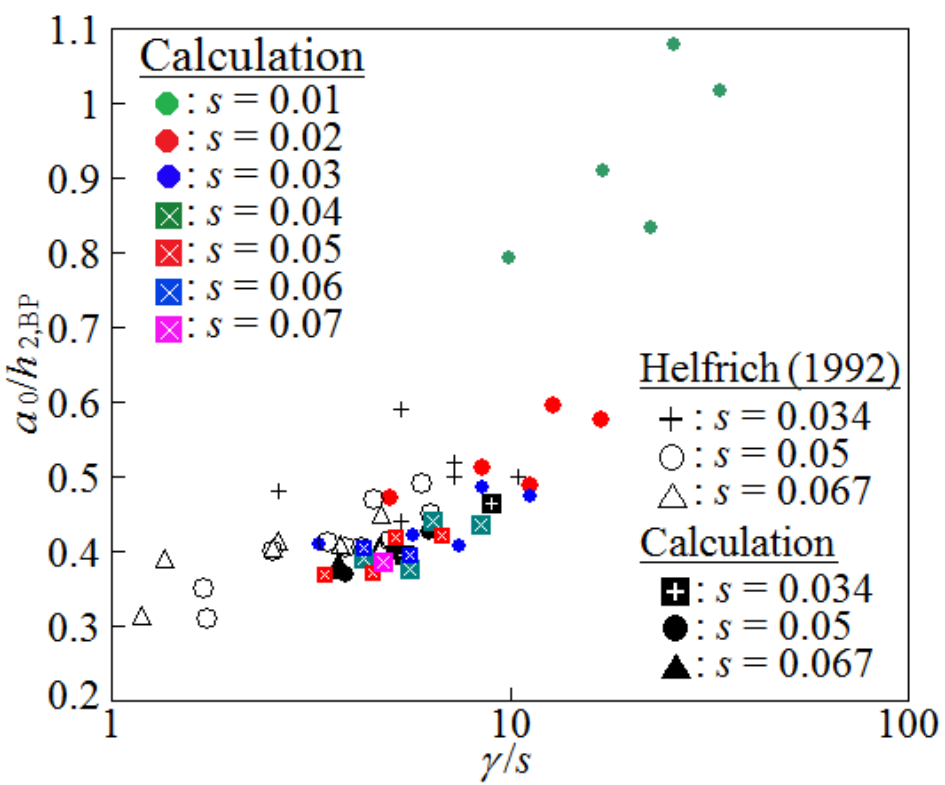

Figure 17. Location of wave-braking points through the present model, where $N=3$, and the experiments by Helfrich (1992).

tank. The internal waves showed remarkable shoaling after the interface touched the critical level. In the lower layer, the horizontal velocity became larger than the local celerity of linear internal waves in shallow water just before the crest peak and the position was defined as the wave-breaking point when the ratio of nonlinear parameter to beach slope, $\gamma / s$, was larger than 3.5. The ratio of initial wave height to wave-breaking depth, $a_{0} / h_{2, \mathrm{BP}}$, became larger as the slope was milder and the wave nonlinearity was stronger. On the mildest slope where the slope was equal to 0.01 the wave height did not increase so much before wave breaking because of the wave dispersion through the longer distance travel, where the energy of internal waves had been provided to the waves in the following wave train. 


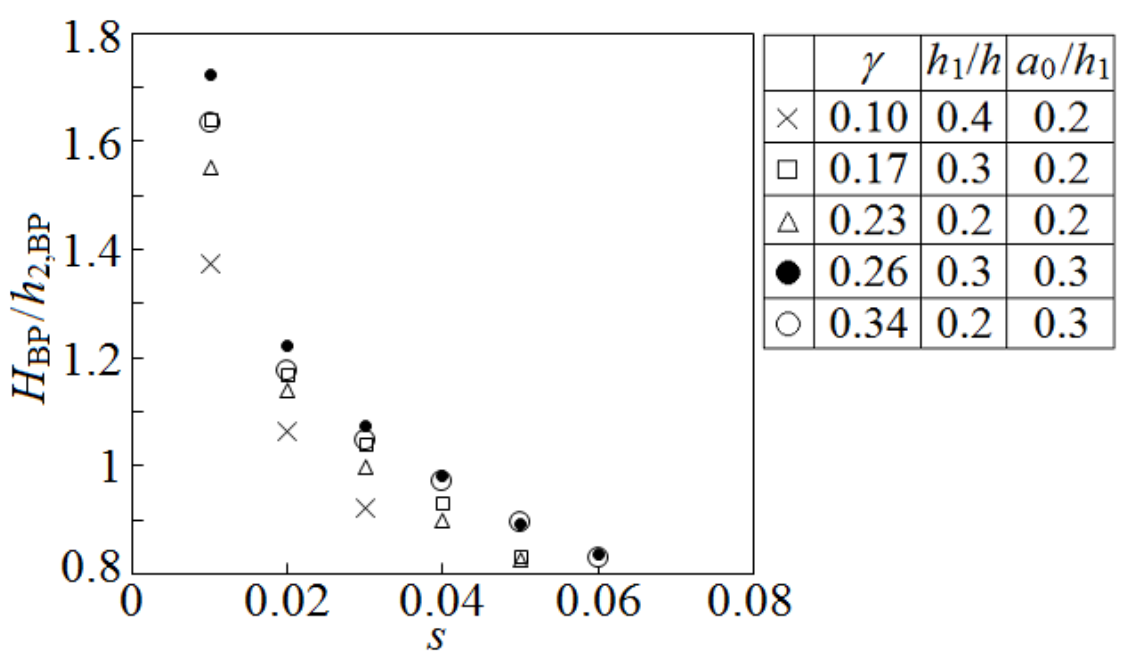

Figure 18. Relation between the beach slope $s$ and the ratio of wave height at the wave-breaking point, $H_{\mathrm{BP}}$, to wave-breaking depth $h_{2, \mathrm{BP}}$.

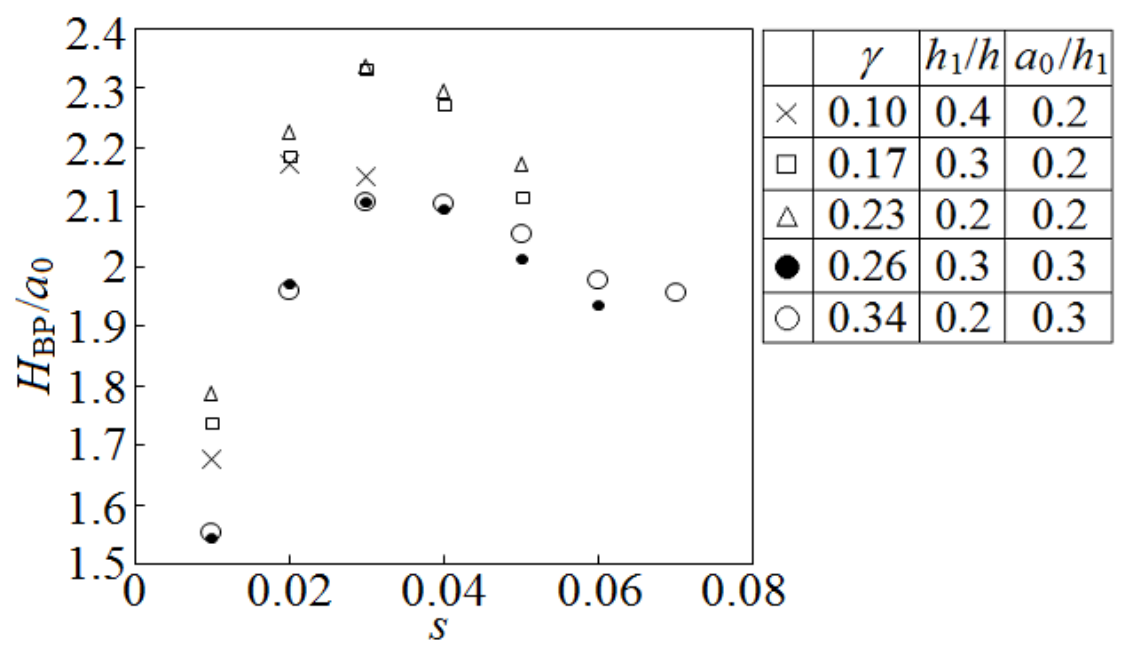

Figure 19. Relation between the beach slope $s$ and the amplification factor of wave height, $H_{\mathrm{BP}} / a_{0}$.

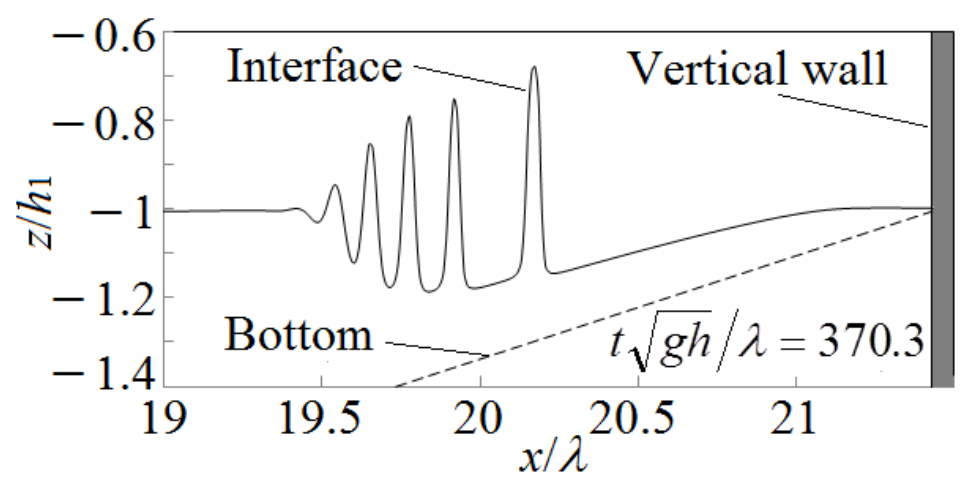

Figure 20. Interface profile at $t^{*}=370.3 \mathrm{~s}\left(\rho_{2} / \rho_{1}=1.02, h_{1} / h=0.2, a_{0} / h_{1}=0.3, s=0.01\right)$. 


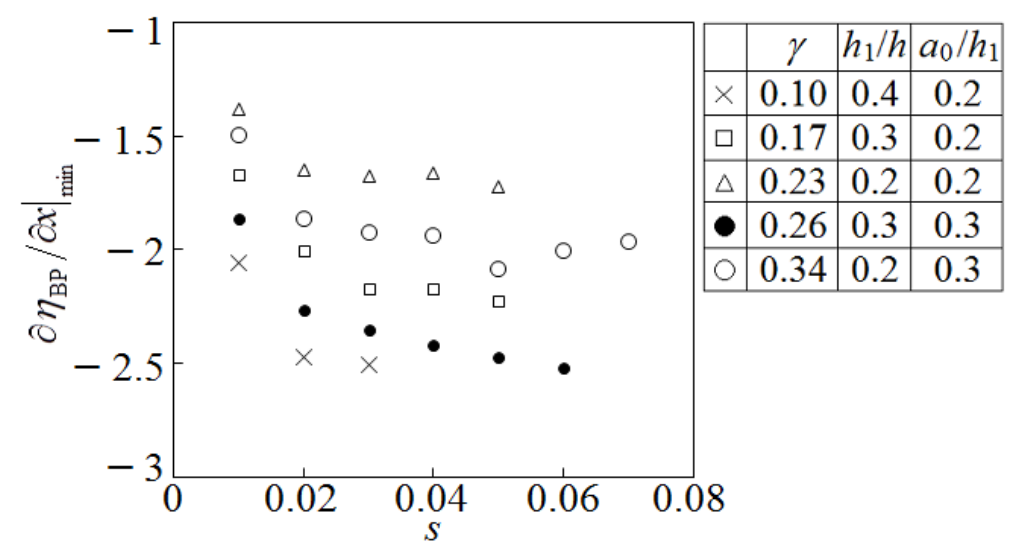

Figure 21. Relation between the beach slope $s$ and the minimum interface gradient of the rear face of the first trough at the wave-breaking point.

\section{REFERENCES}

Aghsaee, P., L. Boegman, and K. G. Lam. 2010. Breaking of shoaling internal solitary waves, The Journal of Fluid Mechanics, 659, 289-317.

Benjamin, T. B. 1966. Internal waves of permanent form in fluids of great depth, The Journal of Fluid Mechanics, 25, 559-592.

Choi, W., and R. Camassa. 1999. Fully nonlinear internal waves in a two-fluid system, The Journal of Fluid Mechanics, 396, 1-36.

Helfrich, K. R. 1992. Internal solitary wave breaking and run-up on a uniform slope, The Journal of Fluid Mechanics, 243, 133-154.

Horn, D. A., L. G. Redekopp, J. Imberger, and G. N. Ivey. 2000. Internal wave evolution in a spacetime varying field, The Journal of Fluid Mechanics, 424, 279-301.

Kakinuma, T. 2001. A set of fully nonlinear equations for surface and internal gravity waves, Proceedings of 5th International Conference on Computer Modelling of Seas and Coastal Regions, WIT Press, 225-234.

Koop, C. G., and G. Butler. 1981. An investigation of internal solitary waves in a two-fluid system, The Journal of Fluid Mechanics, 112, 225-251.

Nakayama, K., and T. Kakinuma. 2010. Internal waves in a two-layer system using fully nonlinear internal-wave equations, International Journal for Numerical Methods in Fluids, 62, 574-590.

Ono, H. 1975. Algebraic solitary waves in stratified fluids, Journal of the Physical Society of Japan, 39, 1082-1091.

Yamashita, K., T. Kakinuma, and K. Nakayama. 2011. Numerical analyses on propagation of nonlinear internal waves, Proceedings of 32nd International Conference on Coastal Engineering, ASCE, waves. 24, 1-15. 